\title{
On controllability for a nondensely defined fractional differential equation with a deviated argument
}

\author{
A. Raheem ${ }^{1}$ - M. Kumar ${ }^{1}$
}

Received: 12 July 2019 / Accepted: 13 October 2019 / Published online: 26 October 2019

(c) The Author(s) 2019

\begin{abstract}
This article deals with a fractional differential equation with a deviated argument defined on a nondense set. A fixed-point theorem and the concept of measure of noncompactness are used to prove the existence of a mild solution. Furthermore, by using the compactness of associated cosine family, we proved that system is approximately controllable and obtains an optimal control which minimizes the performance index. To illustrate the abstract result, we included an example.
\end{abstract}

Keywords Approximate controllability $\cdot$ Optimal control $\cdot$ Deviated argument $\cdot$ Fractional differential equation $\cdot$ Cosine family

Mathematics Subject Classification 93B05 · 49J15 · 34A08 · 47D09 • 47D62 · 34G20 · 34G45 • 47D06 · 47J35

\section{Introduction}

Exact controllability and approximate controllability are some fundamental concepts in control problems. These concepts help to design and analyze the various kinds of control dynamics process in finite- and infinite-dimensional spaces. In the last few decades, control problems have been a research area of great interest. An extensive study on controllability for various kinds of control problems in abstract spaces has been done by many authors [1-15]. In [1], authors proved the approximate controllability for semi-linear noninteger differential equations by using fixed-point technique and semigroup theory of bounded linear operators in a Hilbert space. Das et al. [2] used the concept of measure of noncompactness and the compactness of associated families of linear operators to investigate the sufficient conditions for approximate controllability of a noninteger-order control system with delay depending on state variable. More works on approximate controllability for statedependent noninteger control have been done in [12, 13]. Most of the papers available in the literature deal with densely defined

A. Raheem

araheem.iitk3239@gmail.com

M. Kumar

manojkumar30687@gmail.com

1 Department of Mathematics, Aligarh Muslim University, Aligarh 202002, India systems in abstract spaces. There are only few papers in which authors considered problems involving nondensely defined linear operators. Fu [5] has proved some results on controllability for a nondensely defined abstract differential equations. Other results on controllability for nondensely defined problems have been done in [5, 15, 16]. In [17], authors investigated some sufficient conditions for approximate controllability of delayed semilinear system by using sequence method and $C_{0}$-semigroup of bounded linear operators.

In the last decade, many authors extended the study of approximate controllability to higher order differential equations in abstract spaces [2, 4, 8, 11]. Shukla et al. [4, 8] have studied the second-order semilinear control system and proved that under some assumptions, system is approximately controllable. In [11], stochastic differential equation of order 2 has been studied. The study of controllability to noninteger-order differential equations has been done in [9, $10,18]$. In [10], authors discussed the approximate controllability for a noninteger-order stochastic control system of order $1<\alpha \leq 2$. Few authors [18, 19] investigated the optimal controllability for noninteger-order systems. For more work on controllability of fractional differential equations, we refer [20], and papers cited in this paper.

The present work is motivated by the work of Fu [5], in which authors considered a semilinear control system with linear part is defined on a nondense domain and satisfies the Hille-Yosida condition and established some results 
on controllability. We extended the study of approximate and optimal controllability to a class of nondensely defined fractional differential equations with deviated argument in a Hilbert space by using the measure of noncompactness and Darbo-Sadovskii fixed-point theorem. Consider the following fractional differential equations with deviated argument in a Hilbert space $(Z,\|\cdot\|)$ :

$$
\begin{cases}{ }_{C} D^{\alpha} z(t)=A z(t)+E w(t)+g(t, z(t), z(b(z(t), t))), & 0<t \leq a_{0}, \\ z(0)=z_{0}, & z^{\prime}(0)=z_{1},\end{cases}
$$

where $1<\alpha \leq 2,{ }_{C} D^{\alpha}$, denotes the (Caputo) fractional derivative, $A: D(A) \subset Z \rightarrow Z$ is a nondensely defined linear operator which satisfies the Hille-Yosida condition, $E$ denotes a bounded linear operator defined on $W$ and $w \in L^{2}\left(\left[0, a_{0}\right], W\right)$ denotes the control function, which takes values in a Banach space $W$. The maps $g, b$ are suitably defined functions satisfying some suitable conditions and $z_{0}, z_{1} \in \overline{D(A)}$. Let $I_{0}=\left[0, a_{0}\right]$.

The organization of the rest of the manuscript is as follows. In Section 2, we state some basic concepts and assumptions. In Section 3, first we prove the existence of mild solution, then the approximate and optimal controllability for problem (1) by using a fixed-point theorem and the concept of measure of noncompactness. In the last section to illustrate the abstract result, we included an example.

\section{Preliminaries and assumptions}

In this section, we state some basic definitions, assumptions and notations.

It is well known that if $A$ satisfies Hille-Yosida condition, then $A$ generates an integrated semigroup which is nondegenerate and locally Lipschitz continuous [21, 22].

Define $A_{0}$ on $D\left(A_{0}\right) \subset \overline{D(A)}$ into $\overline{D(A)}$ by

$A_{0} z=A z$,

where

$D\left(A_{0}\right)=\{z \in D(A) \mid A z \in \overline{D(A)}\}$.

Then, $A_{0}$ generates (see [21]), a compact family $\left\{C_{\alpha}(t)\right\}_{t \geq 0}$ of cosines on $\overline{D(A)}$. For $t \geq 0$, we define

$S_{\alpha}(t)=\int_{0}^{t} C_{\alpha}(s) \mathrm{d} s$,

$P_{\alpha}(t)=\frac{1}{\Gamma(\alpha-2)} \int_{0}^{t}(t-s)^{\alpha-2} C_{\alpha}(s) \mathrm{d} s$.

Let
$C\left(I_{0}, \overline{D(A)}\right)=\left\{z: I_{0} \rightarrow \overline{D(A)} \mid z\right.$ is continuous on $\left.I_{0}\right\}$,

$C_{L}\left(I_{0}, \overline{D(A)}\right)=\left\{z \in C\left(I_{0}, \overline{D(A)}\right)\|\| z(t)-z(s) \| \leq L|t-s|\right\}$.

Obviously, $\left(C_{L}\left(I_{0}, \overline{D(A)}\right),\|z\|_{a_{0}}\right)$ is a Banach space, where

$\|z\|_{a_{0}}=\sup _{t \in I_{0}}\|z(t)\|$.

Definition 1 A mild solution of problem (1) is a function $z \in C_{L}\left(I_{0}, \overline{D(A)}\right)$ satisfying the integral equation:

$$
\begin{aligned}
z(t)= & C_{\alpha}(t) z_{0}+S_{\alpha}(t) z_{1} \\
& +\int_{0}^{t} P_{\alpha}(t-s)[g(s, z(s), z(b(z(s), s)))+E w(s)] \mathrm{d} s, \\
& 0 \leq t \leq a_{0} .
\end{aligned}
$$

Next, we define the approximate controllability on a nondensely defined domain.

Definition 2 System (1) is approximately controllable on $I_{0}$, if for every $\epsilon_{0}>0$ and $z_{0}, z_{1}, z_{a_{0}} \in \overline{D(A)}$, there is an admissible control $w \in L^{2}\left(I_{0}, W\right)$ such that the solution $z(t)$ of (1) satisfies

$\left\|z\left(a_{0}\right)-z_{b_{0}}\right\|<\epsilon_{0}$.

Definition 3 The map $Q_{0}: Z_{1} \rightarrow Z_{1}$ is said to be an $\phi$-contraction if there exists a positive constant $k_{0}<1$ such that $\phi\left(Q_{0} C_{0}\right) \leq k_{0} \phi\left(C_{0}\right)$ for any bounded closed subset $C_{0} \subseteq Z_{1}$, where $Z_{1}$ is a Banach space and $\phi$ denotes the Hausdorff measure of noncompactness defined on bounded subsets of $Z_{1}$.

It is convenient to define the operator

$\Omega_{0}^{a_{0}}=\int_{0}^{a_{0}} P_{\alpha}(t-s) E E^{*} P_{\alpha}^{*}(t-s) \mathrm{d} s$,

where $E^{*}$ and $P_{\alpha}^{*}$ are the adjoint operators of $E$ and $P_{\alpha}$, respectively.

For $\delta>0$, let

$R\left(\delta, \Omega_{0}^{a_{0}}\right)=\left(\delta I+\Omega_{0}^{a_{0}}\right)^{-1}$

and

$$
\begin{aligned}
q(z(t))= & z_{a_{0}}-C_{\alpha}\left(a_{0}\right) z_{0}-S_{\alpha}\left(a_{0}\right) z_{1} \\
& -\int_{0}^{a_{0}} P_{\alpha}\left(a_{0}-s\right) g(s, z(s), b(z(s), s)) \mathrm{d} s,
\end{aligned}
$$

then we have

$w_{z}(t)=E^{*} P_{\alpha}^{*}\left(a_{0}-t\right) R\left(\delta, \Omega_{0}^{a_{0}}\right) q(z(t))$. 
Lemma 4 If the map $Q_{0}: D\left(Q_{0}\right) \subseteq Z_{1} \rightarrow Z_{1}$ is a Lipschitz continuous with constant $k_{0}$, then $\phi\left(Q C_{0}\right) \leq k_{0} \phi\left(C_{0}\right)$, for any bounded set $C_{0}$.

Lemma 5 [23] (Darbo-Sadovskii fixed-point theorem) $A$ $\phi$-contraction continuous map $Q_{0}: W_{0} \rightarrow W_{0}$, defined on a convex, closed and bounded set $W_{0} \subseteq Z_{1}$, has at least one fixed point in $W_{0}$.

We consider the following assumptions:

(H1) Map g satisfies:

(1) $\left\|g\left(t_{1}, z_{1}, \tilde{z}_{1}\right)-g\left(t_{2}, z_{2}, \tilde{z}_{2}\right)\right\|_{a_{0}} \leq L_{g}\left[\left|t_{1}-t_{2}\right|+\| z_{1}\right.$ $\left.-z_{2}\left\|_{a_{0}}+\right\| \tilde{z}_{1}-\tilde{z}_{2} \|_{a_{0}}\right]$

(2) $\|g(t, z, \tilde{z})\| \leq G_{0}$,

for some $L_{g}, G_{0}>0$, and for all $t, t_{1}, t_{2} \in I_{0}$, $z, z_{1}, z_{2} \in C\left(I_{0}, \overline{D(A)}\right)$ and $\tilde{z}, \tilde{z}_{1}, \tilde{z}_{2} \in C_{L}\left(I_{0}, \overline{D(A)}\right)$.

(H2) There exists a constant $L_{b}>0$ such that $\|b(z(t), t)-b(\tilde{z}(t), t)\| \leq L_{b}\|z(t)-\tilde{z}(t)\|$.

(H3) There exist constants $M_{1}>0, M_{2}>0$ and $r_{1}>0$ such that

$\left\|C_{\alpha}(t)\right\| \leq M_{1}, \quad\left\|S_{\alpha}(t)\right\| \leq M_{2}, \quad\left\|P_{\alpha}(t)\right\| \leq r_{1}, \quad t \in I_{0}$.

(H4) There exists a constant $M_{3}>0$ such that $\|E\| \leq M_{3}$.

Lemma 6 Under assumptions (H1)-(H4), there exists $K_{w}>0$ s.t. $\left\|w_{z}(t)\right\| \leq K_{w}$.

Proof Using the definition of control function $w_{z}(t)$, and (H4), we have

$\left\|w_{z}(t)\right\|=\left\|E^{*}\right\|\left\|P_{\alpha}^{*}\left(a_{0}-t\right)\right\|\left\|R\left(\delta, \Omega_{0}^{a_{0}}\right)\right\|\|q(z(t))\|$

$$
\leq \frac{M_{3} r_{1}}{\delta}\|q(z(t))\| \text {. }
$$

In view of assumptions (H1)-(H3), we can find a constant $K_{w}>0$, such that

$\left\|w_{z}(t)\right\| \leq K_{w}$.

\section{Main results}

Theorem 1 If ( $\mathrm{H} 1)-(\mathrm{H} 4)$ hold, then for every $z_{0}, z_{1}, z_{a_{0}} \in \overline{D(A)}$, system (1) has a mild solution on $I_{0}$, if $r_{1} a_{0} L_{g}\left(1+L L_{b}\right)\left[1+\frac{r_{1}^{2} a_{0} M_{3}^{2}}{\delta}\right]<\frac{1}{2}$.

Proof To show that system (1) has a mild solution, we need to show that, there exists $z \in C_{L}\left(I_{0}, \overline{D(A)}\right)$, such that

$$
\begin{aligned}
z(t)= & C_{\alpha}(t) z_{0}+S_{\alpha}(t) z_{1} \\
& +\int_{0}^{t} P_{\alpha}(t-s)\left[g(s, z(s), z(b(z(s), s)))+E w_{z}(s)\right] \mathrm{d} s \\
& 0 \leq t \leq a_{0} .
\end{aligned}
$$

We define the operator $\tilde{G}_{\delta}$ on $C_{L}\left(I_{0}, \overline{D(A)}\right)$, by

$$
\begin{aligned}
\left(\tilde{G}_{\delta} z\right)(t)= & C_{\alpha}(t) z_{0}+S_{\alpha}(t) z_{1} \\
& +\int_{0}^{t} P_{\alpha}(t-s)\left[g(s, z(s), z(b(z(s), s)))+E w_{z}(s)\right] \mathrm{d} s, \\
& 0 \leq t \leq a_{0} .
\end{aligned}
$$

Let

$B_{r_{0}}=\left\{z \in C_{L}\left(I_{0}, \overline{D(A)}\right) \mid z(0)=z_{0}, z^{\prime}(0)=z_{1},\|z\|_{a_{0}} \leq r_{0}\right\}$.

If $z(t)$ satisfies (2), then $z(t)$ can be written as $z(t)=x_{0}(t)+x(t)$, where

$$
\begin{aligned}
x_{0}(t)= & C_{\alpha}(t) z_{0}+S_{\alpha}(t) z_{1} \\
x(t)= & \int_{0}^{t} P_{\alpha}(t-s)\left[g \left(s, x_{0}(s)+x(s), x_{0}\left(b\left(x_{0}(s)+x(s), s\right)\right)\right.\right. \\
& \left.\left.+x\left(b\left(x_{0}(s)+x(s), s\right)\right)\right)+E w_{x_{0}+x}(s)\right] \mathrm{d} s .
\end{aligned}
$$

Define $\tilde{F}_{\delta}: C_{L}\left(I_{0}, \overline{D(A)}\right) \rightarrow C_{L}\left(I_{0}, \overline{D(A)}\right)$, by

$$
\begin{aligned}
\left(\tilde{F}_{\delta} x\right)(t)= & \int_{0}^{t} P_{\alpha}(t-s)\left[g \left(s, x_{0}(s)+x(s), x_{0}\left(b\left(x_{0}(s)+x(s), s\right)\right)\right.\right. \\
& \left.\left.+x\left(b\left(x_{0}(s)+x(s), s\right)\right)\right)+E w_{x_{0}+x}(s)\right] \mathrm{d} s .
\end{aligned}
$$

Obviously, map $\tilde{G}_{\delta}$ has a fixed point if and only if $\tilde{F}_{\delta}$ has a fixed point.

For $r>0$, we define

$C_{r}=\left\{z \in C_{L}\left(I_{0}, \overline{D(A)}\right): z(0)=0, z^{\prime}(0)=0,\|z\|_{a_{0}} \leq r\right\}$.

Clearly, $\tilde{F}_{\delta}$ is well defined on $C_{r}$ which is a closed convex set in $C_{L}\left(I_{0} ; \overline{D(A)}\right)$. 
To show that $\tilde{F}_{\delta}$ has a fixed point, we use Darbo-Sadovskii fixed-point theorem. For this, we need to follow the two steps:

Step 1: There exists $r>0$, s.t. $\tilde{F}_{\delta}\left(C_{r}\right) \subseteq C_{r}$. Let $x \in C_{r}$. Then, using (H2), we have

$$
\begin{aligned}
\left\|\left(\tilde{F}_{\delta} x\right)(t)\right\| \leq & r_{1} \int_{0}^{t}\left[\| g\left(s, x_{0}(s)+x(s), x_{0}\left(b\left(x_{0}(s)+x(s), s\right)\right)\right.\right. \\
& \left.\left.+x\left(b\left(x_{0}(s)+x(s), s\right)\right)\right)+E w_{x_{0}+x}(s) \|\right] \mathrm{d} s .
\end{aligned}
$$

Using (H1), (H4) and Lemma 6, we get

$\left\|\left(\tilde{F}_{\delta} x\right)(t)\right\| \leq r_{1}\left(G_{0} a_{0}+M_{3} K_{w}\right)$.

We can choose $r>0$, sufficiently large such that

$\left\|\left(\tilde{F}_{\delta} x\right)(t)\right\| \leq r$.

Thus, there exists $r>0$ such that

$\tilde{F}\left(C_{r}\right) \subseteq C_{r}$.

Step 2: $\tilde{F}_{\delta}$ is a $\phi$-contraction map. For this, first, we need to show that $\tilde{F}_{\delta}$ is Lipschitz continuous. Using (H3) and (H4), we have

$$
\begin{aligned}
& \left\|\left(\tilde{F}_{\delta} x_{1}\right)(t)-\left(\tilde{F}_{\delta} x_{2}\right)(t)\right\| \\
& \leq r_{1} \int_{0}^{t} \| g\left(s, x_{0}(s)+x_{1}(s), x_{0}\left(b\left(x_{0}(s)+x_{1}(s), s\right)\right)\right. \\
& \left.\quad+x_{1}\left(b\left(x_{0}(s)+x_{1}(s), s\right)\right)\right) \\
& \quad-g\left(s, x_{0}(s)+x_{2}(s), x_{0}\left(b\left(x_{0}(s)+x_{2}(s), s\right)\right)\right. \\
& \left.\quad+x_{2}\left(b\left(x_{0}(s)+x_{2}(s), s\right)\right)\right) \| \mathrm{d} s \\
& \quad+r_{1} M_{3} \int_{0}^{t}\left\|w_{x_{0}+x_{1}}(s)-w_{x_{0}+x_{2}}(s)\right\| \mathrm{d} s .
\end{aligned}
$$

Using (H1), (H2) and (H3), we get

$$
\begin{aligned}
\| g(s, & x_{0}(s)+x_{1}(s), x_{0}\left(b\left(x_{0}(s)+x_{1}(s), s\right)\right) \\
& \left.+x_{1}\left(b\left(x_{0}(s)+x_{1}(s), s\right)\right)\right) \\
& -g\left(s, x_{0}(s)+x_{2}(s), x_{0}\left(b\left(x_{0}(s)+x_{2}(s), s\right)\right)\right. \\
& \left.+x_{2}\left(b\left(x_{0}(s)+x_{2}(s), s\right)\right)\right) \| \\
\leq & L_{g}\left[\left\|x_{1}(s)-x_{2}(s)\right\|+L\left\|b\left(x_{0}(s)+x_{1}(s), s\right)-b\left(x_{0}(s)+x_{2}(s), s\right)\right\|\right. \\
& \left.+\left\|x_{1}\left(b\left(x_{0}(s)+x_{1}(s), s\right)\right)-x_{2}\left(b\left(x_{0}(s)+x_{2}(s), s\right)\right)\right\|\right] \\
\leq & L_{g}\left[\left\|x_{1}(s)-x_{2}(s)\right\|+L L_{b}\left\|x_{1}(s)-x_{2}(s)\right\|\right. \\
& +\left\|x_{1}\left(b\left(x_{0}(s)+x_{1}(s), s\right)\right)-x_{2}\left(b\left(x_{0}(s)+x_{1}(s), s\right)\right)\right\| \\
& \left.+\left\|x_{2}\left(b\left(x_{0}(s)+x_{1}(s), s\right)\right)-x_{2}\left(b\left(x_{0}(s)+x_{2}(s), s\right)\right)\right\|\right] \\
\leq & 2 L_{g}\left[1+L L_{b}\right]\left\|x_{1}-x_{2}\right\|_{a_{0}} .
\end{aligned}
$$

and

$$
\begin{aligned}
& \left\|w_{x_{0}+x_{1}}(s)-w_{x_{0}+x_{2}}(s)\right\| \\
& \quad \leq \frac{M_{3} r_{1}}{\delta}\left\|q\left(\left(x_{0}+x_{1}\right)(s)\right)-q\left(\left(x_{0}+x_{2}\right)(s)\right)\right\| . \\
& \quad \leq \frac{2 M_{3} r_{1}^{2}}{\delta} a_{0} L_{g}\left(1+L L_{b}\right)\left\|x_{1}-x_{2}\right\|_{a_{0}} .
\end{aligned}
$$

From (3), we have

$$
\begin{aligned}
& \left\|\left(\tilde{F}_{\delta} x_{1}\right)(t)-\left(\tilde{F}_{\delta} x_{2}\right)(t)\right\| \\
& \quad \leq 2 r_{1} a_{0} L_{g}\left(1+L L_{b}\right)\left[1+\frac{r_{1}^{2} a_{0} M_{3}^{2}}{\delta}\right]\left\|x_{1}-x_{2}\right\|_{a_{0}} .
\end{aligned}
$$

Thus, $\tilde{F}_{\delta}$ is Lipschitz continuous. By using Lemma 4 , we have

$\phi\left(\tilde{F}_{\delta} C_{r}\right) \leq 2 r_{1} a_{0} L_{g}\left(1+L L_{b}\right)\left[1+\frac{r_{1}^{2} a_{0} M_{3}^{2}}{\delta}\right] \phi\left(C_{r}\right)$.

Since $2 r_{1} a_{0} L_{g}\left(1+L L_{b}\right)\left[1+\frac{r_{1}^{2} a_{0} M_{3}^{2}}{\delta}\right]<1, \tilde{F}_{\delta}$ is a $\phi$-contraction, by using Lemma $5, \tilde{F}_{\delta}$ has a fixed point and consequently $\tilde{G}_{\delta}$ has a fixed point, say $z_{\delta}$.

Theorem 2 Under all the assumptions of Theorem 1, system (1) is approximately controllable on $I_{0}$.

Proof The fixed point $z_{\delta}$ obtained in Theorem 1 is given by:

$$
\begin{aligned}
z_{\delta}(t)= & C_{\alpha}(t) z_{0}+S_{\alpha}(t) z_{1} \\
& +\int_{0}^{t} P_{\alpha}(t-s)\left[g\left(s, z_{\delta}(s), z_{\delta}\left(b\left(z_{\delta}(s), s\right)\right)\right)\right. \\
& \left.+E w_{z_{\delta}}(s)\right] \mathrm{d} s,
\end{aligned}
$$

where

$$
w_{z_{\delta}}(t)=D^{*} P_{\alpha}^{*}\left(a_{0}-t\right) R\left(\delta, \Omega_{0}^{a_{0}}\right) q\left(z_{\delta}(t)\right),
$$

and

$$
\begin{aligned}
q\left(z_{\delta}(t)\right)= & z_{a_{0}}-C_{\alpha}\left(a_{0}\right) z_{0}-S_{\alpha}\left(a_{0}\right) z_{1} \\
& -\int_{0}^{a_{0}} P_{\alpha}\left(a_{0}-s\right) g\left(s, z_{\delta}(s), b\left(z_{\delta}(s), s\right)\right) \mathrm{d} s .
\end{aligned}
$$


Thus, we have

$$
\begin{aligned}
z_{\delta}\left(a_{0}\right)-z_{a_{0}} & =-q\left(z_{\delta}(t)\right)+\Omega_{0}^{a_{0}} R\left(\delta, \Omega_{0}^{a_{0}}\right) q\left(z_{\delta}(t)\right) \\
& =-\left(\delta I+\Omega_{0}^{a_{0}}\right) R\left(\delta, \Omega_{0}^{a_{0}}\right) q\left(z_{\delta}(t)\right)+\Omega_{0}^{a_{0}} R\left(\delta, \Omega_{0}^{a_{0}}\right) q\left(z_{\delta}(t)\right) \\
& =-\delta R\left(\delta, \Omega_{0}^{a_{0}}\right) q\left(z_{\delta}(t)\right) .
\end{aligned}
$$

Now

$\int_{0}^{a_{0}}\left\|g\left(s, z_{\delta}(s), b\left(z_{\delta}(s), s\right)\right)\right\|^{2} \mathrm{~d} s \leq G_{0}^{2} a_{0}$.

Thus, the sequence $\left\{g\left(t, z_{\delta}(t), b\left(z_{\delta}(t), t\right)\right)\right\}$ is bounded in the Hilbert space $L^{2}\left(I_{0}, Z\right)$. Therefore, there exists a weakly convergent subsequence $\left\{g\left(t, z_{\delta}(t), b\left(z_{\delta}(t), t\right)\right)\right\}$ (again denoted by same notation) converging to $v(\cdot) \in L^{2}\left(I_{0}, Z\right)$. Let

$\mu=z_{a_{0}}-C_{\alpha}\left(a_{0}\right) z_{0}-S_{\alpha}\left(a_{0}\right) z_{1}-\int_{0}^{a_{0}} \tilde{P}_{\alpha}\left(a_{0}-s\right) v(s) \mathrm{d} s$.

Thus,

$\left\|q\left(z_{\delta}(t)\right)-\mu\right\| \leq\left\|\int_{0}^{a_{0}} \tilde{P}_{\alpha}\left(a_{0}-s\right)\left[g\left(s, z_{\delta}(s), b\left(z_{\delta}(s), s\right)\right)-v(s)\right] \mathrm{d} s\right\|$.

By using the compactness of $P_{\alpha}(t)$, the Ascola-Arzela theorem, and following the idea used in [1], one can prove that the mapping

$z(t) \rightarrow \int_{0}^{a_{0}} P_{\alpha}(t-s) z(s) \mathrm{d} s$

is compact. Therefore, we have

$\left\|q\left(z_{\delta}(t)\right)-\mu\right\| \rightarrow 0 \quad$ as $\delta \rightarrow 0$.

From (4), we have

$$
\begin{aligned}
\left\|z_{\delta}\left(a_{0}\right)-z_{0}\right\| & =\left\|\delta R\left(\delta, \Omega_{0}^{a_{0}}\right) q\left(z_{\delta}(t)\right)\right\| \\
& \leq\left\|\delta R\left(\delta, \Omega_{0}^{a_{0}}\right)\right\|\left\|q\left(z_{\delta}(t)\right)-\mu\right\|+\left\|\delta R\left(\delta, \Omega_{0}^{a_{0}}\right) \mu\right\| .
\end{aligned}
$$

This implies that $\left\|z_{\delta}\left(a_{0}\right)-z_{0}\right\| \rightarrow 0$ as $\delta \rightarrow 0$. Thus, system (1) is approximately controllable on $I_{0}$.

Next, we discuss the optimal controllability of problem (1). In order to proceed further, we define performance index

$\tilde{J}(w)=\int_{0}^{a_{0}} \tilde{F}(t, z(t), z(b(z(t), t)), w(t)) \mathrm{d} t$, where $\tilde{F}$ is a functional defined on $I_{0} \times C\left(I_{0}, Z\right) \times C_{L}\left(I_{0}, Z\right) \times W_{a d}$ and $W_{a d}$ denotes the set of all admissible control and consequently is closed and convex in $L^{2}\left(I_{0}, W\right)$.

Theorem 3 Under all the assumptions of Theorem 1, there exists an optimal control of problem (1) if $r_{1} a_{0} L_{g}\left(2+L L_{b}\right)<1$.

Proof To complete the proof of the theorem, it is sufficient to find a control $w^{0} \in L^{2}\left(I_{0}, W\right)$ that minimizes the performance index $\tilde{J}(w)$.

If $\inf \left\{\tilde{J}(w) \mid w \in W_{a d}\right\}=\infty$, then result trivially holds.

If $\inf \left\{\tilde{J}(w) \mid w \in W_{a d}\right\}=\epsilon_{0}<\infty$, then there exists a sequence $\left\{w^{n}\right\}$ in $W_{a d}$ such that $\tilde{J}\left(w^{n}\right) \rightarrow \epsilon_{0}$. As $W_{a d}$ is a closed and convex subset of $L_{2}\left(I_{0}, W\right)$, the sequence $\left\{w^{n}\right\}$ has a weakly convergent subsequence $\left\{w^{m}\right\}$ converging to $w^{0} \in W_{a d}$. Using Theorem 1 , for each $w^{m} \in W_{a d}$, there exists a mild solution $z^{m}$ of (1) satisfying:

$$
\begin{aligned}
z^{m}(t)= & C_{\alpha}(t) z_{0}+S_{\alpha}(t) z_{1} \\
& +\int_{0}^{t} P_{\alpha}(t-s)\left[g\left(s, z^{m}(s), z^{m}\left(b\left(z^{m}(s), s\right)\right)\right)\right. \\
& \left.+E w^{m}(s)\right] \mathrm{d} s, \quad 0 \leq t \leq a_{0} .
\end{aligned}
$$

Similarly, corresponding to $w^{0}$, there exists a mild solution $z^{0}$ of (1) satisfying:

$$
\begin{aligned}
z^{0}(t)= & C_{\alpha}(t) z_{0}+S_{\alpha}(t) z_{1} \\
& +\int_{0}^{t} P_{\alpha}(t-s)\left[g\left(s, z^{0}(s), z^{0}\left(b\left(z^{0}(s), s\right)\right)\right)\right. \\
& \left.+E w^{0}(s)\right] \mathrm{d} s, \quad 0 \leq t \leq a_{0}
\end{aligned}
$$

Using (H3), for $t \in\left[0, a_{0}\right]$, we have

$$
\begin{aligned}
\| z^{m}(t) & -z^{0}(t) \| \\
\leq & r_{1} \int_{0}^{t}\left[\left\|g\left(s, z^{m}, z^{m}\left(b\left(z^{m}(s), s\right)\right)\right)-g\left(s, z^{0}, z^{0}\left(b\left(z^{0}(s), s\right)\right)\right)\right\|\right. \\
& \left.+\left\|E w^{n}(s)-E w^{0}(s)\right\|\right] \mathrm{d} s .
\end{aligned}
$$

Using (H1), (H2), we get

$$
\begin{aligned}
&\left\|g\left(s, z^{m}(s), z^{m}\left(b\left(z^{m}(s), s\right)\right)\right)-g\left(s, z^{0}(s), z^{0}\left(b\left(z^{0}(s), s\right)\right)\right)\right\| \\
& \leq L_{g}\left[\left\|z^{m}(s)-z^{0}(s)\right\|+\left\|z^{m}\left(b\left(z^{m}(s), s\right)\right)-z^{0}\left(b\left(z^{0}(s), s\right)\right)\right\|\right] \\
& \leq L_{g}\left[\left\|z^{m}(s)-z^{0}(s)\right\|+\left\|z^{m}\left(b\left(z^{m}(s), s\right)\right)-z^{m}\left(b\left(z^{0}(s), s\right)\right)\right\|\right. \\
&\left.\quad+\left\|z^{m}\left(b\left(z^{0}(s), s\right)\right)-z^{0}\left(b\left(z^{0}(s), s\right)\right)\right\|\right] \\
& \leq L_{g}\left(2+L L_{b}\right)\left\|z^{m}-z^{0}\right\|_{a_{0}} .
\end{aligned}
$$


From (5), we have

$$
\begin{aligned}
& \left\|z^{m}(t)-z^{0}(t)\right\| \\
& \quad \leq r_{1}\left[L_{g}\left(2+L L_{b}\right) a_{0}\left\|z^{m}-z^{0}\right\|_{a_{0}}+\int_{0}^{t}\left\|E w^{m}(s)-E w^{0}(s)\right\| \mathrm{d} s\right] \\
& \quad \leq r_{1} a_{0}\left[L_{g}\left(2+L L_{b}\right)\left\|z^{m}-z^{0}\right\|_{a_{0}}+\left\|E w^{m}-E w^{0}\right\|_{a_{0}}\right] .
\end{aligned}
$$

Since $r_{1} a_{0} L_{g}\left(2+L L_{b}\right)<1$ and $\left\|E w^{m}-E w^{0}\right\|_{a_{0}} \rightarrow 0$, we conclude that $z^{m} \rightarrow z^{0}$.

Applying Balder's theorem, we get

$$
\begin{aligned}
\epsilon_{0} & =\lim _{n \rightarrow \infty} \int_{0}^{a_{0}} \tilde{F}\left(t, z^{m}(t), z^{m}\left(b\left(z^{m}(t), t\right)\right), w^{m}(t)\right) \mathrm{d} t \\
& \leq \int_{0}^{a_{0}} \tilde{F}\left(t, z^{0}(t), z^{0}\left(b\left(z^{0}(t), 0\right)\right), w^{0}(t)\right) \mathrm{d} t \\
& =\tilde{J}\left(w^{0}\right) \geq \epsilon_{0} .
\end{aligned}
$$

This shows that $\tilde{J}\left(w^{0}\right)=\epsilon_{0}$, i.e., $\tilde{J}$ attains its minimum value at $w^{0} \in L^{2}\left(I_{0}, W\right)$.

\section{Application}

Consider the following example:

$$
\left\{\begin{array}{l}
{ }_{C} D^{\frac{3}{2}} z(y, s)-\frac{\partial^{2}}{\partial y^{2}} z(y, s)=v_{0}(y, s)+g_{1}(y, z(y, s))+g_{2}(s, y, z(y, s)), \\
y \in(0, \pi) \quad s \in\left(0, a_{0}\right], \\
z(0)=z(\pi)=0, \\
z(y, 0)=z_{0}(y), \quad \frac{\partial z}{\partial s}(y, 0)=z_{1}(y), \quad y \in(0, \pi),
\end{array}\right.
$$

where

$g_{1}(y, z(y, s))=\int_{0}^{y} K(y, x) z\left(y, a_{1} h(t)|z(x, t)|\right) \mathrm{d} x$,

and the function $g_{2}: R_{+} \times[0,1] \times R \rightarrow R$ is locally Hölder continuous in $t$, locally Lipschitz continuous in $z$, uniformly in $y$ and measurable in $y$. Let $I_{0}=\left[0, a_{0}\right]$.

Choose $Z=C([0, \pi], R)$ and the operator $A$ defined by $A z=z^{\prime \prime}$ with domain

$D(A)=\left\{z(\cdot) \in Z \mid z^{\prime \prime} \in Z, z(0)=z(\pi)=0\right\}$.

Obviously, $\overline{D(A)} \neq Z$. Thus, $A$ is nondensely defined on $Z$.

Also $\rho(A) \supseteq(0,+\infty),\left\|(\lambda I-A)^{-1}\right\| \leq \frac{1}{\lambda}$ for $\lambda>0$. This shows that $A$ satisfies the Hille-Yosida on $Z$. Therefore, $A$ generates a compact $C_{0}$-semigroup $\{T(t)\}$ on $\overline{D(A)}$ and consequently $A$ generates a compact cosine family $\left\{C_{\alpha}(t)\right\}_{t \geq 0}$ on $\overline{D(A)}$.
Let $C\left(I_{0}, \overline{D(A)}\right)$ denote the set of all continuous functions on $I_{0}$, and

$C_{L}\left(I_{0}, \overline{D(A)}\right)=\left\{z \in C\left(I_{0}, \overline{D(A)}\right)\left|\left\|z\left(s_{1}\right)-z\left(s_{2}\right)\right\| \leq L\right| s_{1}-s_{2} \mid\right\}$.

We define $g: I_{0} \times C\left(I_{0}, \overline{D(A)}\right) \times C_{L}\left(I_{0}, \overline{D(A)}\right) \rightarrow X$, by

$g(s, z, \phi)(y)=g_{1}(y, \phi)+g_{2}(s, y, z)$,

and $(E v)(s)(y)=v_{0}(s, y), z(s)(y)=z(y, s)$; then, abstract formulation of problem (6) is

$$
\begin{aligned}
{ }_{C} D^{\frac{3}{2}} z(s) & =A z(s)+E v(s)+g(s, z(s), b(z(s), s)) \\
z(0) & =z_{0}, \quad \frac{\partial z}{\partial s}(0)=z_{1} .
\end{aligned}
$$

We can easily prove that all assumptions of Theorem 2 are satisfied; therefore by using theorem, we conclude that system (6) is approximately controllability on $I_{0}$.

Define performance index

$\tilde{J}(w)=\int_{0}^{a_{0}}\left(\|z(t)\|^{2}+\|w(t)\|^{2}\right) \mathrm{d} t$.

It can be easily checked that all the assumptions of Theorem 3 are satisfied; therefore, by using Theorem 3 , we find an admissible control $w_{0}$ that minimizes $\tilde{J}(w)$.

Open Access This article is distributed under the terms of the Creative Commons Attribution 4.0 International License (http://creativeco mmons.org/licenses/by/4.0/), which permits unrestricted use, distribution, and reproduction in any medium, provided you give appropriate credit to the original author(s) and the source, provide a link to the Creative Commons license, and indicate if changes were made.

\section{References}

1. Sakthivel, R., Ren, Y., Mahmudov, N.I.: On the approximate controllability of semilinear fractional differential systems. Comput. Math. Appl. 62, 1451-1459 (2011)

2. Das, S., Pandey, D.N., Sukavanam, N.: Approximate controllability of a second order natural differential equation with state dependent delay. Differ. Equ. Dyn. Syst. 24(2), 201-214 (2016)

3. Chen, L., Li, G.: Approximate controllability of impulsive differential equations with nonlocal condition. Int. J. Nonlinear Sci. 10(4), 438-446 (2010)

4. Shukla, A., Sukavanam, N., Pandey, D.N., Arora, U.: Approximate controllability of second order semilinear control system. Circuits Syst. Signal Process. 35, 3339-3354 (2016)

5. Fu, X.: Controllability of non-densely defined functional differential systems in abstract space. Appl. Math. Lett. 19, 369-377 (2006)

6. Wang, J., Fan, Z., Zhou, Y.: Nonlocal controllability of semilinear dynamic systems with fractional derivative in Banach spaces. J. Optim. Theory Appl. 154, 292-302 (2012) 
7. Fan, Z.: Approximate controllability of fractional differential equations via resolvent operators. Adv. Differ. Equ. 2014(54), $1-11(2014)$

8. Shukla, A., Sukavanam, N., Pandey, D.N.: Approximate controllability of second order semilinear stochastic system with nonlocal conditions. Ann. Univ. Ferrara 61, 355-366 (2015)

9. Jeet, K., Bahuguna, D.: Approximate controllability of nonlocal neutral fractional integro-differential equations with finite delay. J. Dyn. Control Syst. 22, 485-504 (2016)

10. Shukla, A., Sukavanam, N., Pandey, D.N.: Approximate controllability of fractional semilinear stochastic system of order $\alpha \in(1,2]$. J. Dyn. Control Syst. 23, 679-691 (2017)

11. Das, S., Pandey, D.N., Sukavanam, N.: Approximate controllability of a second-order neutral stochastic differential equation with state-dependent delay. Nonlinear Anal. Model. Control 21(6), 751-769 (2016)

12. Rathinasamy, S., Yong, R.: Approximate controllability of fractional differential equations with state-dependent delay. Results Math. 63, 949-963 (2013)

13. Das, S., Pandey, D.N., Sukavanam, N.: Existence of solution and approximate controllability of a second order neutral stochastic differential equation with state dependent delay. Acta Math. Sci. 36B(5), 1509-1523 (2016)

14. Górniewicz, L., Ntouyas, S.K., O'Regan, D.: Existence and controllability results for first and second order functional semilinear differential inclusions with nonlocal conditions. Numer. Funct. Anal. Optim. 28(1-2), 53-82 (2007)

15. Adimy, M., Ezzinbi, K.: A class of linear partial neutral functional differential equations with nondense domain. J. Differ. Equ. 147, 285-332 (1998)
16. Thieme, H.R.: "Integrated semigroups" and integrated solutions to abstract Cauchy problems. J. Math. Anal. Appl. 152, 416-447 (1990)

17. Shukla, A., Sukavanam, N., Pandey, D.N.: Approximate controllability of semilinear system with state delay using sequence method. J. Frankl. Inst. 352, 5380-5392 (2015)

18. Ozdemir, N., Karadeniz, D., Inskender, B.B.: Fractional optimal control problem of a distributed system in cylindrical coordinates. Phys. Lett. A 373(2), 221-226 (2009)

19. Wang, J.R., Wei, W., Zhou, J.: Fractional finite time delay evolution systems and optimal controls in infinite dimensional spaces. J. Dyn. Control Syst. 17(4), 515-535 (2011)

20. Kexue, L., Jigen, P., Jinghuai, G.: Controllability of nonlocal fractional differential systems of order $\alpha \in(1,2]$ in Banach spaces. Rep. Math. Phys. 71(1), 33-43 (2013)

21. Bazhlekova, E.: Fractional Evolution Equations in Bannch Spaces. University Press Facilities, Eindhoven University of Technology, Eindhoven (2001)

22. Pazy, A.: Semigroups of Linear Operators and Applications to Partial Differential Equations. Springer, Berlin (1983)

23. Banas, J., Goebel, K.: Measure of Noncompactness in Banach spaces. Marcel Dekker, New York (1980)

Publisher's Note Springer Nature remains neutral with regard to jurisdictional claims in published maps and institutional affiliations 